\section{Adaptação cultural do Chronic Liver Disease Questionnaire (CLDQ) para população brasileira}

\author{
Cross-cultural adaptation of the Chronic Liver \\ Disease Questionnaire (CLDQ) to the Brazilian \\ population
}

\author{
Samantha Mucci 1 \\ Vanessa de Albuquerque Citero 1 \\ Adriano Miziara Gonzalez 2 \\ Mario Alfredo De Marco 1 \\ Luiz Antonio Nogueira-Martins 1
}

1 Departamento de

Psiquiatria, Universidade Federal de São Paulo, São Paulo, Brasil.

2 Departamento de

Gastroenterologia Cirúrgica, Universidade Federal de São

Paulo, São Paulo, Brasil

Correspondência

S. Mucci

Departamento de Psiquiatria, Universidade Federal de São Paulo.

Rua Borges Lagoa 570, 9o andar, São Paulo, SP 04038-020, Brasil.

sammucci@gmail.com

\begin{abstract}
The aims of this study were the English-to-Portuguese translation and cross-cultural adaptation of the Chronic Liver Disease Questionnaire (CLDQ) for use in Brazil. The instrument was translated from the original language, English, to Portuguese by the authors, and was subsequently reviewed and evaluated as to the degree of difficulty of the translation and equivalence, by bilingual translators. The questionnaire was then applied to 20 randomly selected patients with chronic liver disease. Patients had no difficulty understanding the questionnaire and considered all the questions applicable. The cultural equivalence of the CLDQ was demonstrated, without requiring changes in the original translation. The translation into Portuguese and cross-cultural adaptation of the CLDQ successfully completed this important stage for its validation and use in Brazil.
\end{abstract}

Liver Diseases; Questionnaires; Cross-Cultural Comparison

\section{Introdução}

A doença hepática crônica é a 9a causa de morte no mundo e é responsável pela incapacitação de uma parcela economicamente ativa da população 1. Segundo Organização Mundial da Saúde (OMS) 2, existem atualmente 350 milhões de pessoas portadoras de doença hepática crônica pelo vírus da hepatite (B e C). Diante deste contingente torna-se fundamental cuidar da qualidade de vida destes pacientes 1 .

O conceito de qualidade de vida definido pela OMS é “ a percepção do indivíduo de sua posição na vida, no contexto da cultura esistema de valores nos quais ele vive e em relação aos seus objetivos, expectativas, padrões e preocupações" 3 (p. 179).

Qualidade de vida relacionada à saúde é um conceito subjetivo e multidimensional, influenciado por fatores sócio-econômicos, idade, sexo, presença de doença e tratamento, medindo o impacto de uma doença na vida de um indivíduo ${ }^{4}$.

A qualidade de vida dos pacientes com doença hepática crônica varia de acordo com as mudanças em seu estado clínico 5 , visto que, com a piora da hepatopatia, muitos pacientes perdem sua autonomia, sentem-se cansados, com desconfortos abdominais, insônia e mudanças de humor.

Houve, na última década, uma proliferação de instrumentos de mensuração de qualidade de vida 6 , tanto genéricos (uso na população geral, podem comparar doenças crônicas diferentes) 
quanto específicos (uso em populações caracterizadas por uma determinada doença, identificam as particularidades da situação) 5 .

São três os instrumentos específicos utilizados na avaliação de qualidade de vida de pacientes portadores de hepatopatia crônica:

- Hepatitis Quality of Life Questionnaire (HQOLQ): não é aplicável em pacientes em estágio de cirrose avançado. Foi validado somente para uso em pacientes com hepatite tipo C 7 .

- Liver Disease Quality of Life Instrument (LDQOL 1.0): instrumento muito longo utilizado apenas em pacientes em estágio avançado de cirrose 8 . Foi traduzido e validado para população brasileira 9 .

- Chronic Liver Disease Questionnaire (CLDQ): único instrumento desenvolvido e validado para todas as etiologias e graus de gravidade da doença hepática 5 .

O presente estudo realizou a tradução para a língua portuguesa e a adaptação transcultural no Brasil do CLDQ, para posteriormente ser realizada a validação do instrumento.

\section{Métodos}

A autorização para a tradução foi concedida pelos autores do instrumento e o projeto de pesquisa foi aprovado pelo Comitê de Ética em Pesquisa da Universidade Federal de São Paulo (UNIFESP), onde foi realizado. A UNIFESP, localizada na capital, presta assistência à saúde a pacientes da capital e de outros estados.

\section{Pacientes}

A maioria dos estudos de tradução e adaptação transcultural utiliza amostra de 10 pacientes, porém alguns estudos utilizam amostra de 20 pacientes, abrangendo diversidade maior de pessoas, aumentando a possibilidade de compreensão do instrumento 9,10 .

Nesse estudo, a amostra foi composta de 20 pacientes portadores de doença hepática crônica do Serviço de Transplante de Fígado do Hospital São Paulo (UNIFESP), selecionados de acordo com o agendamento de consultas do ambulatório, no período de janeiro a fevereiro de 2005. Os pacientes eram todos maiores de 18 anos, com doença diagnosticada por exames clínicos e laboratoriais.

Os critérios de exclusão foram incapacidade cognitiva e estar em tratamento anti-retroviral. Nenhum paciente foi excluído e todos foram informados sobre os objetivos da pesquisa, assinando termo de consentimento.

\section{Instrumentos}

Foi aplicado um questionário sócio-demográfico e clínico e utilizada a escala Child-TurcottePugh 11 para avaliação da gravidade da doença.

O CLDQ 5 é um instrumento curto e autoaplicável. Constituído por 29 questões distribuídas em seis domínios, cada questão apresenta sete níveis de resposta: de 0 (todo tempo) a 6 (nunca). O CLDQ apresenta um escore por domínio e um total, com caráter multidimensional, avaliando a percepção geral da qualidade de vida e enfatizando os sintomas da doença hepática. O escore em cada domínio é obtido pela soma das respostas e dividido pelo número de questões compreendidas. O escore total é obtido pela soma dos domínios e dividido por 6. Os seis domínios (sintomas abdominais, fadiga, sintomas sistêmicos, atividade, função emocional e preocupação) foram identificados pela análise de principais componentes, com proporção de variação de 0,056 a 0,224 5 .

\section{Procedimentos}

A tradução e adaptação transcultural foi realizada de forma padronizada 9,10,12, de acordo com a seqüência:

1) Tradução inicial: os itens da versão em inglês do CLDQ (Tabela 1) foram traduzidos para a língua portuguesa por dois profissionais da saúde bilíngües, independentes e cientes do objetivo desta pesquisa. Estes realizaram tradução conceitual e não estritamente literária. As traduções foram comparadas pelos tradutores e por um dos autores, sendo constituída uma tradução consensual;

2) Avaliação da tradução por retro-tradução: a tradução consensual foi vertida para o inglês por dois tradutores bilíngües, independentes, cientes do objetivo da pesquisa e diferentes dos dois anteriores. Posteriormente, foi realizada a comparação dessas versões com o instrumento original em inglês e as avaliações da tradução indicaram equivalência e reconciliação dos itens, com equivalência semântica entre as traduções. Para a realização da equivalência idiomática e equivalência conceitual, o comitê de especialistas (dois gastrocirurgiões, uma enfermeira e uma psicóloga) sugeriu a inclusão de explicação em um item (colocada entre parênteses) para melhor compreensão. O comitê, também, discutiu as questões em relação a sua validade de face e de conteúdo 12;

3) Avaliação da equivalência cultural (piloto/ pré-teste): com finalidade de identificar as questões não compreendidas por nossa população, foi colocada ao lado de cada questão a opção 
Perguntas e escala de respostas do Chronic Liver Disease Questionnaire (CLDQ).

\section{The Chronic Liver Disease Questionnaire (CLDO)}

This questionnaire is designed to find out how you have been feeling during the last two weeks. You will be asked about your symptoms related to your

liver disease, how you have been affected in doing activities, and how your mood has been. Please complete all of the questions and select only one response for each question.

1. How much of the time during the last two weeks have you been troubled by a feeling of abdominal bloating?

2. How much of the time have you been tired or fatigued during the last two weeks?

3. How much of the time during the last 2 weeks have you experienced bodily pain?

4. How often during the last two weeks have you felt sleepy during the day?

5. How much of the time during the last two weeks have you experienced abdominal pain?

6. How much of the time during the last two weeks has shortness of breath been a problem for you in your daily activities?

7. How much of the time during the last two weeks have you not been able to eat as much as you would like?

8. How much of the time in the last two weeks have you been bothered by having decreased strength?

9. How often during last 2 weeks have you had trouble lifting or carrying heavy objects?

10. How often during the last two weeks have you felt anxious?

11. How often during the last 2 weeks have you felt a decreased level of energy?

12. How much of the time during the last two weeks have you felt unhappy?

13. How often during the last two weeks have you felt drowsy?

14. How much of the time during the last two weeks have you been bothered by a limitation of your diet?

15. How often during the last two weeks have you been irritable?

16. How much of the time during the last two weeks have you had difficulty sleeping at night?

17. How much of the time during the last two weeks have you been troubled by a feeling of abdominal discomfort?

18. How much of the time during the last two weeks have you been worried about the impact your liver disease has on your family?

19. How much of the time during the last two weeks have you had mood swings?

20. How much of the time during the last two weeks have you been unable to fall asleep at night?

21. How often during the last two weeks have you had muscle cramps?

22. How much of the time during the last two weeks have you been worried that your symptoms will develop into major problems?

23. How much of the time during the last two weeks have you had a dry mouth?

24. How much of the time during the last two weeks have you felt depressed?

25. How much of the time during the last two weeks have you been worried about your condition getting worse?

26. How much of the time during the last two weeks have you had problems concentrating?

27. How much of the time have you been troubled by itching during the last two weeks?

28. How much of the time during the last two weeks have you been worried about never feeling any better?

29. How much of the time during the last two weeks have you been concerned about the availability of a liver if you need a liver transplant?

Answers

\footnotetext{
1. All of the time

2. Most of the time

3. A good bit of the time

4. Some of the time

5. A little of the time

6. Hardly any of the time

7. None of the time
}

Fonte: Younossi et al. 5.

“não aplicável” (Tabela 2). A aplicação foi realizada por dois entrevistadores treinados, os dados obtidos foram submetidos à análise exploratória com descrição das freqüências de resposta.

\section{Resultados}

Os pacientes entrevistados eram predominantemente do sexo feminino (65\%), viviam com companheiro/a (45\%), idade média de 48 anos (DP = 11 anos, variação de 18 a 65 anos), e escolaridade média de 8 anos ( $\mathrm{DP}=4$, variação de 1 a 15 
Tabela 2

Chronic Liver Disease Questionnaire (CLDQ)-BR piloto (como o instrumento foi utilizado na tradução e adaptação cultural).

Este questionário avalia como você vem se sentindo nas últimas duas semanas. Você será questionado sobre os sintomas relacionados à sua doença hepática, como vem sendo afetado em suas atividades e como está o seu humor. Por favor, responda a todas as questões, selecionando apenas uma alternativa para cada questão:

1. Por quanto tempo, nas duas últimas semanas, você foi afetado por uma sensação de empachamento abdominal (estufamento abdominal)?

2. Por quanto tempo, nas duas últimas semanas, você se sentiu cansado ou com fadiga?

3. Por quanto tempo, nas duas últimas semanas, você sentiu dores no corpo?

4. Por quanto tempo, nas duas últimas semanas, você sentiu sonolência durante o dia?

5. Por quanto tempo, nas duas últimas semanas, você sentiu dores abdominais?

6. Por quanto tempo, nas duas últimas semanas, você ficou ofegante?

7. Por quanto tempo, nas duas últimas semanas, você não conseguiu comer o quanto gostaria de ter comido?

8. Por quanto tempo, nas duas últimas semanas, você se sentiu incomodado nas suas atividades por ter a força diminuída?

9. Com que freqüência, nas duas últimas semanas, você teve dificuldades para levantar ou carregar objetos pesados?

10. Com que freqüência, nas duas últimas semanas, você se sentiu ansioso?

11. Com que freqüência, nas duas últimas semanas, você sentiu uma diminuição de sua energia?

12. Por quanto tempo, nas duas últimas semanas, você se sentiu infeliz?

13. Com que freqüência, nas duas últimas semanas, você se sentiu sonolento?

14. Por quanto tempo, nas duas últimas semanas, você se sentiu incomodado pelas limitações de sua dieta?

15. Com que freqüência, nas duas últimas semanas, você se sentiu irritado?

16. Por quanto tempo, nas duas últimas semanas, você teve dificuldades para dormir à noite?

17. Por quanto tempo, nas duas últimas semanas, você foi incomodado por um desconforto abdominal?

18. Por quanto tempo, nas duas últimas semanas, você ficou preocupado com o impacto que a sua doença hepática tem sobre sua família?

19. Por quanto tempo, nas duas últimas semanas, você teve mudanças de humor?

20. Por quanto tempo, nas duas últimas semanas, você foi incapaz de adormecer à noite?

21. Com que freqüência, nas duas últimas semanas, você teve cãibras musculares?

22. Por quanto tempo, nas duas últimas semanas, você se preocupou com o fato de que seus sintomas possam se tornar problemas mais graves?

23. Por quanto tempo, nas duas últimas semanas, você teve boca seca?

24. Por quanto tempo, nas duas últimas semanas, você se sentiu deprimido?

25. Por quanto tempo, nas duas últimas semanas, você se preocupou com a possibilidade de sua doença se agravar?

26. Por quanto tempo, nas duas últimas semanas, você teve problemas de concentração?

27. Por quanto tempo, nas duas últimas semanas, você teve problemas com coceiras?

28. Por quanto tempo, nas duas últimas semanas, você se preocupou com a possibilidade de nunca vir a melhorar?

29. Por quanto tempo, nas duas últimas semanas, você se preocupou com a disponibilidade de um fígado para transplante, caso venha a precisar de um?

\section{Respostas}

1. Todo tempo

2. A maior parte do tempo

3. Uma parte do tempo

4. Alguma parte do tempo

5. Uma pequena parte do tempo

6. Quase nada

7. Nunca

Não aplicável

anos de estudo), 25\% estavam desempregados e $35 \%$ eram aposentados pela doença. A distribuição por diagnóstico e sintomatologia hepática é apresentada na Tabela 3 .

Nenhuma das 29 questões foi considerada "não aplicável” pelos pacientes, sendo a equivalência cultural do CLDQ realizada sem a necessidade de uma segunda etapa de aplicação do instrumento.
A maior parte dos pacientes (80\%) necessitou de auxílio do entrevistador para ler as perguntas, porém, não foi observada dificuldade dos pacientes em escolher as respostas. O tempo médio de aplicação do CLDQ foi de 15 minutos $( \pm 5)$. 
Distribuição das características clínicas da população.

\begin{tabular}{|c|c|}
\hline Características clínicas & Freqüência (\%) \\
\hline \multicolumn{2}{|l|}{ Diagnóstico de doença hepática } \\
\hline Viral tipo C & 25 \\
\hline Alcoólica & 25 \\
\hline Viral tipo C + alcoólica & 20 \\
\hline Auto-imune & 5 \\
\hline Outros (viral tipo B/hipertensão portal/colangite esclerosante/doença de Wilson) & 25 \\
\hline \multicolumn{2}{|l|}{ Sintomas } \\
\hline Ascite & 65 \\
\hline Icterícia & 65 \\
\hline Hemorragia digestiva alta & 65 \\
\hline Fadiga & 50 \\
\hline Spiders & 25 \\
\hline Eritema palmar & 10 \\
\hline \multicolumn{2}{|l|}{ Gravidade de doença (escala de Child-Pugh) } \\
\hline A & 30 \\
\hline$B$ & 65 \\
\hline C & 5 \\
\hline Tempo de doença hepática (variação de 6 a 240 meses) & Mediana $=36$ meses \\
\hline
\end{tabular}

\section{Discussão}

No presente estudo, pudemos verificar que as etapas de tradução e retro-tradução não apresentaram dificuldades e a equivalência semântica e conceitual foram facilmente obtidas. $\mathrm{O}$ comitê de especialistas colaborou para a obtenção da equivalência cultural sugerindo a inserção da explicação entre parênteses pelo fato de os itens originais não se enquadrarem no linguajar habitual da população brasileira. Na primeira questão “abdominal bloating”, foi na versão final do CLDQ traduzido como empachamento abdominal e o comitê sugeriu a inserção ao lado: (estufamento). Assim como, discutiram sobre o instrumento e o que se propõe medir e concluíram que o CLDQ contempla e é capaz de medir a qualidade de vida geral e por domínios do paciente portador de doença hepática crônica.

Podemos considerar como limitação de nosso estudo, a necessidade da presença de entrevistador pela maioria dos pacientes. Tentamos minimizar os possíveis vieses do entrevistador, oferecendo treinamento rigoroso que acarretou um aumento de custo e talvez de tempo durante aplicação do instrumento.

O CLDQ foi traduzido e validado em outros países (Alemanha 13, Tailândia 14, Espanha 15, Itália 16) e apresentou boas propriedades psicométricas em todas as versões. Estes dados corro- boram a possibilidade de o instrumento ser adequado para validação na população brasileira.

\section{Conclusão}

A tradução e adaptação transcultural do CLDQ para a população brasileira foram concluídas de acordo com a metodologia adequada. As etapas de validação de constructo (convergente e divergente), de critério e confiabilidade do CLDQ no Brasil estão sendo concluídas. 


\section{Resumo}

Nesse estudo realizaram-se a tradução para o português e a adaptação cultural do instrumento Chronic Liver Disease Questionnaire (CLDQ) para uso no Brasil. O instrumento foi traduzido da versão original (inglês) para a língua portuguesa pelos autores $e$, posteriormente, revisado e avaliado quanto ao grau de dificuldade das traduções e equivalência por tradutores bilíngües. O instrumento foi, então, aplicado em 20 pacientes com hepatopatia crônica selecionados aleatoriamente. Não houve dificuldade na compreensão do instrumento, todas as questões foram consideradas aplicáveis pelos pacientes, e a equivalência cultural do CLDQ foi demonstrada sem que mudanças na tradução original precisassem ser feitas. A tradução e a adaptação cultural do CLDQ para o português, no Brasil, foram realizadas, tendo sido cumprida esta importante etapa para sua validação e utilização em nosso meio.

Hepatopatias; Questionários; Comparação Transcultural

\section{Colaboradores}

S. Mucci participou do delineamento do estudo, da revisão da literatura, da elaboração da pesquisa, da tradução do questionário do inglês para o português, da análise e discussão dos resultados e da redação do artigo. V. A. Citero e L. A. Nogueira-Martins contribuíram com a elaboração da pesquisa, análise dos resultados, discussão dos resultados e na redação do artigo. A. M. Gonzalez e M. A. De Marco participaram da discussão dos resultados e da composição do artigo.

\section{Referências}

1. Younossi ZM. Epidemiology of alcoholic liver disease. Clin Liver Dis 1998; 2:661-71.

2. World Health Organization. Hepatitis B. http:// www.who.int/mediacentre/factsheets/fs204/en/ index.html (acessado em Set/2009).

3. Fleck MPA, Louzada S, Xavier M, Chachamovich E, Vieira G, Santos L, et al. Aplicação da versão em português do instrumento WHOQOL-bref. Rev Saúde Pública 2000; 34:178-83.

4. Glise H, Wilklund I Health-related quality of life and gastrointestinal disease. J Gastroenterol Hepatol 2002; 17 Suppl:S72-S84.

5. Younossi ZM, Guyatt G, Kiwi M, Boparai N, King D. Development of a disease specific questionnaire to measure health related quality of life in patients with chronic liver disease. Gut 1999; 45:295-300.
6. Seidl EMF, Zannon CMLC. Qualidade de vida e saúde: aspectos conceituais e metodológicos. Cad Saúde Pública 2004; 20:580-8.

7. Ware JE, Bayliss MS, Mannocchia M, Davis GL; The International Hepatitis Interventional Therapy Group. Health-related quality of life in chronic hepatitis C: impact of disease and treatment response. Hepatology 1999; 30:550-5.

8. Gralnek IM, Hays RD, Kilbourne A, Rosen HR, Keefe EB, Artinian L, et al. Development and evaluation of the Liver Disease Quality of Life Instrument in persons with advanced, chronic liver disease - the LDQOL 1.0. Am J Gastroenterol 2000; 95:3552-65. 
9. Teixeira MCD, Ribeiro MFS, Strauss E. A new in sight into differences among non-cirrhotic patients using the Liver Disease Quality of Life Instrument (LDQOL). Ann Hepatol 2005; 4:264-71.

10. Guillemin F, Bombardier C, Bearton D. Cross-cultural adaptation of health-related quality of life measures: literature review and proposed guidelines. J Clin Epidemiol 1993; 46:1417-32.

11. Child CG, Turcotte JG. Surgery and portal hypertension: major problems. Clin Surg 1964; 1:1-85.

12. Viana HB, Madruga VA. Diretrizes para adaptação cultural de escalas psicométricas. Lecturas, Educación Física y Deportes 2008; 116:1-8.

13. Häuser W, Schnur M, Steder-Neukamm U, Muthny FA, Grandt D. Validation of the German version of the Chronic Liver Disease Questionnaire. Eur J Gastroenterol Hepatol 2004; 16:599-606.
14. Sobhonslidsuk A, Silpatik C, Kongsakon R, Satipornkul P, Sripetch C. Chronic Liver Disease Questionnaire: translation and validation in Thais. World J Gastroenterol 2004; 10:1954-7.

15. Ferrer M, Córdoba J, Garin O, Olivé G, Flavià M Vargas V, et al. Validity of the Spanish version of the Chronic Liver Disease Questionnaire (CLDQ) as a standard outcome for quality of life assessment. Liver Transpl 2006; 12:22-3.

16. Rucci P, Taliani G, Cirrincione L, Alberti A, Bartolozzi D, Caporaso N, et al. Validity and reliability of the Italian version of the Chronic Liver Disease Questionnaire (CLDQ-I) for the assessment of health-related quality of life. Dig Liver Dis 2005; 37:850-60.

Recebido em 15/Mar/2009

Versão final reapresentada em 17/Set/2009

Aprovado em 30/Set/2009 